\title{
Designing the height of the first profile of the marshalling hump
}

\author{
Khabibulla Turanov ${ }^{1, *}$, Andrej Gordienko², Shukhrat Saidivaliev ${ }^{3}$, and Shukhrat \\ Djabborov $^{3}$ \\ ${ }^{1}$ Tashkent State Technical University named Islam Karimov, 10000, University Str, 2, Tashkent. \\ Uzbekistan \\ ${ }^{2}$ Ural State University of Railway Transport, 620034, Kolmogorova st., 66, Yekaterinburg, Russia \\ ${ }^{3}$ Tashkent Railway Engineering Institute, 100167, Temirylchilar 1- tor kycha, 1, Tashkent, \\ Uzbekistan
}

\begin{abstract}
The height of the high-speed section of the sorting slide. Design of high-speed section of the marshalling hump. To prove mathematically the absence of theoretical basis of the formula by which the height of the first profile section of the marshalling hump is calculated in current method of calculation of marshalling humps. The paper uses the theorem on the change of kinetic energy for a non-free material point in the final form, known from theoretical mechanics. The results of the research revealed that the formulas by which the height of the first profile section of the marshalling hump is calculated contradict the theorem on the change of kinetic energy for a non-free material point in the final form. The results of the research can be used in the processing of the normative-technical document on the design of hump devices on railways and making adjustments to the dynamics of rolling the car in textbooks for universities of railway transport. The results of the calculations proved the incorrectness of the derivation of the formula for calculating the height of the first profile section of the marshalling hump in the normative-technical document "Rules and standards for the design of sorting devices on the railways with gauge $1520 \mathrm{~mm}$.
\end{abstract}

\section{Introduction}

Continuing to discuss the correctness of the theoretical base of the current calculation methods of hump yards [1 - 18], we note that it is still the subject of discussion not become the theoretical assumptions of the mathematical model formula (8) in the fifth counterexample [12]. It is known that when designing a sorting slide, the height of the first profile section (from the top of the slide to the first arrow zone or the first brake position (1TP)) is calculated by the formula (see formula (8) in [12]):

\footnotetext{
* Corresponding author: khturanov@yandex.ru
} 


$$
h_{1}^{\max }=\frac{v_{\mathrm{in}}^{2}-v_{0}^{2}}{2 g_{o}^{\prime}}+h_{\mathrm{sp} 1}+h_{\mathrm{ac} 1},
$$

where $\boldsymbol{v}_{\text {in }}$ - maximum permissible speed input of uncoupling [accurately, single wagon] $\left[\boldsymbol{v}_{\mathrm{rm}}\right]$ on wagon retarding mechanisms [12], i.e. $\boldsymbol{v}_{\mathrm{in}}=\left[\boldsymbol{v}_{\mathrm{rm}}\right], \mathrm{m} / \mathrm{s}$;

$\boldsymbol{v}_{0}$ - the greatest initial velocity sliding of «very good free axle (GFA)», since $\boldsymbol{G}=908$ $\mathrm{kN}$ accept 1.7 to $2.5 \mathrm{~m} / \mathrm{s}), \mathrm{m} / \mathrm{s}$;

$g_{0}^{\prime}-$ acceleration of gravity taking into account the inertia of the rotating masses GFA, $\mathrm{m} / \mathrm{s}^{2}$

$\boldsymbol{h}_{\text {sp1 }}$ и $\boldsymbol{h}_{\text {ac1 }}$ - losses of specific energy at overcoming of the basic specific resistance to movement and resistance from arrows and curves within advance section $\boldsymbol{l}_{1}$, e.m.h (the energy meter of height).

It should taken into account [12] that the profile height $\boldsymbol{h}_{\mathbf{1}}$ of advance hump section (i.e. from the top of the hump (TH) to the point in the middle 1BP) can be determined from the input condition of the calculated free axle (wagon) he second retarder of the first braking position (1BP) the first wheel pair of the calculation free axle, «good axle (GA)» under favorable conditions of rolling with the maximum allowable for the adopted type of retarder entry speed $\boldsymbol{v}_{\text {in }}=7 \mathrm{~m} / \mathrm{s}$.

In the first term of the formula (1) the rate of entry $\boldsymbol{v}_{\text {in }}$ can be determined by the formula (see formula (3) in [12]) only taking into account the initial velocity $\boldsymbol{v}_{\mathbf{b e g}}=\boldsymbol{v}_{\text {in }}=\boldsymbol{v}_{\mathbf{0}}$, derived for a perfect connection):

$$
v_{\text {in }}=\sqrt{2 \cdot g_{o}^{\prime} \cdot h_{\mathrm{ac}}+v_{o}^{2}},
$$

where $\boldsymbol{v}_{\mathbf{0}}=\boldsymbol{v}_{\mathbf{o d}}-$ rating speed of detaching, e.g., $\boldsymbol{v}_{\mathbf{o d}}=1.4 \mathrm{~m} / \mathrm{s}$ for hump of largecapacity.

Note that if formula (2) is correct, then in formula (1) the first term will be found by formula (3) in [15] only taking into account the initial velocity $\boldsymbol{v}_{\mathbf{b e g}}=\boldsymbol{v}_{\mathbf{i n}}=\boldsymbol{v}_{\mathbf{0}}$, derived for a perfect connection.

It should be noted that in [12] to determine the required height of hump $\boldsymbol{h}_{\text {hump }}$ the kinetic sum is found $\boldsymbol{E}_{\mathbf{0}}$ and potential $\boldsymbol{E}_{\mathbf{p}}$ wagon energies (see formulas (4) and (5) in [16]), $\mathrm{kJ}$

$$
E_{0}=M \frac{v_{0}^{2}}{2} \rho \text { и } E_{P}=g M h_{\mathrm{hump}}
$$

given the fact that they $\boldsymbol{M}$ - mass of the wagon, $\mathrm{t} ; \boldsymbol{v}_{\mathbf{0}}$ - initial speed, $\mathrm{m} / \mathrm{s} ; \boldsymbol{\rho}$ - coefficient that takes into account the correction for the rotation of wheel pairs; $\mathbf{g}=9.81$ - gravitational acceleration, $\mathrm{m} / \mathrm{s}^{2} ; \boldsymbol{h}_{\text {hump }}$ - required height of the hump, $\mathrm{m}$.

Total energy (kinetic $\boldsymbol{E}_{\mathbf{0}}$ and potential $\boldsymbol{E}_{\mathbf{p}}$ ) equated to the work of the component of the gravity projection $(\boldsymbol{G} \sin \psi)$ of a moving wagon, which is taken to be equal to the sum of the works of the forces of resistance of every kind $\boldsymbol{A}_{\mathbf{r}, \mathbf{c}, \mathbf{s}}$ :

$$
M \frac{v_{0}^{2}}{2} \rho+g M h_{\mathrm{hump}}=A_{\mathrm{r}, \mathrm{c}, \mathrm{s}}
$$

where

$$
A_{\mathrm{r}, \mathrm{c}, \mathrm{s}}=A_{r}+A_{c}+A_{s}
$$

given what they indicate: $\boldsymbol{A}_{\mathbf{r}}$ - the work of the forces of resistance in the movement of the wagon at a distance between the top of the hump (TH) and a control point $(100 \mathrm{~m}$ 
further from TH), kJ; $\boldsymbol{A}_{\mathbf{c}}$ - the work of the resistance forces during the passage of the curve section of the track, kJ; $\boldsymbol{A}_{\mathbf{s}}$ - work of resistance forces at impact of wheels of wheel pairs of the wagon about switches, kJ.

Work of resistance forces $\boldsymbol{A}_{\mathrm{r}}$ (see formula (1) в [16]), kJ:

$$
A_{r}=g M \frac{r_{m} \cdot l}{1000}
$$

where $\boldsymbol{r}_{\mathbf{m}}$ - coefficient taking into account rolling resistance of wagon wheels, $\mathrm{N} / \mathrm{kN} ; \boldsymbol{l}-$ the distance between the vertex and the considered reference point, $\mathrm{m}$.

The work of the resistance forces during the passage of the curve section of the track $\boldsymbol{A}_{\mathbf{c}}$ (see formula (2) in [16]), kJ:

$$
A_{c}=g M \frac{\sum r_{c} \cdot l_{c}}{1000}=g M \frac{c \cdot \sum \alpha_{c}}{1000}
$$

where $\boldsymbol{r}_{\mathbf{c}}-$ coefficient taking into account the resistivity of the curve, $\mathrm{N} / \mathrm{kN} ; \boldsymbol{l}_{\mathbf{c}}-$ track length in curve, $\mathrm{m} ; c \cdot \sum \alpha_{c}$ - work of relative resistance of a curve of a site of track, $\mathrm{N} / \mathrm{kN}$; c - coefficient taking into account the specific resistance of the wagon on the curve, $\mathrm{N} / \mathrm{kN}$; $\sum \alpha_{c}$ - the sum of the central angles of rotation of the curve of the track section, deg.

Work of resistance forces at impact of wheels of wheel pairs of the wagon about switches $\boldsymbol{A}_{\text {s }}$ (see formula (3) in [16]), kJ:

$$
A_{s}=g M \frac{20 \cdot n}{1000},
$$

where $\boldsymbol{n}$ - the number track switches on the studied section of the track profile.

Substituting formulas (6) - (8) in (4) obtain equality (see equality (7) in [16]):

$$
M \frac{v_{0}^{2}}{2} \rho+g M h_{\text {hump }}=g M \frac{r_{m} \cdot l}{1000}+g M \frac{c \cdot \sum \alpha_{c}}{1000}+g M \frac{20 \cdot n}{1000} .
$$

After elementary mathematical transformations of the last equation to obtain a formula

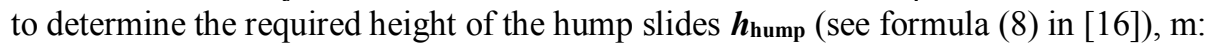

$$
h_{\text {hump }}=\frac{r_{m} \cdot l}{1000}+\frac{c \cdot \sum \alpha_{c}}{1000}+\frac{20 \cdot n}{1000}-\frac{v_{0}^{2}}{2 g^{\prime \prime}}
$$

where $g^{\prime}$ - acceleration of gravity taking into account the inertia of the rotating parts of the wagon, $\mathrm{m} / \mathrm{s}^{2}$.

In [16] there is a formula by which the speed of the wagon is determined $\boldsymbol{v}_{2}$ from the hump crest with an initial velocity $\boldsymbol{v}_{\mathbf{1}}=\boldsymbol{v} \mathbf{0}, \mathrm{m} / \mathrm{s}$ :

$$
v_{2}=\sqrt{v_{1}^{2}+\frac{2 g^{\prime}(\mathrm{s}-\mathrm{r}) l}{1000}}
$$

where $\boldsymbol{s}$ - profile grade of track, \% ; r - coefficient, taking into account the resistivity of the movement of the car, $\mathrm{N} / \mathrm{kN}$. Hence it is clear that in $\left[16, \%_{0}=\mathrm{N} / \mathrm{kN}\right.$, which is unacceptable.

In [16] the energy heights between the switch and the considered point of the slide profile are presented in the form, $\mathrm{m}$ :

$$
\frac{v_{i n}^{2}}{2 g^{\prime}}+\frac{s \cdot l}{1000}=\frac{r_{m} \cdot l}{1000}+\frac{c \cdot \sum \alpha}{1000} .
$$


Hence the speed of the wagon is determined $\boldsymbol{\nu}_{\mathbf{H}}$ between the switch and the considered point of the slide profile (see formula (15) in [16]), $\mathrm{m} / \mathrm{s}$ :

$$
v_{i n}=\sqrt{\frac{2 g^{\prime}}{1000}\left[(\mathrm{~s}-\mathrm{r}) l_{t}+c \cdot \sum \alpha\right]}
$$

where $\boldsymbol{l}_{\mathbf{t}}-$ the length of the path between the switch and the considered point, $\mathrm{m}$.

It is well known [6, 19] that for a system with ideal connections and/or for a conservative system, the total energy $\left(\boldsymbol{E}_{\mathbf{0}}+\boldsymbol{E}_{\mathbf{p}}\right)$ remains constant (see p. 293 in [19]):

$$
M \frac{v_{0}^{2}}{2} \rho+g M h_{\text {hump }}=\text { const, }
$$

i.e., the kinetic energy of the car $\boldsymbol{E}_{\mathbf{0}}$ with mass $\boldsymbol{M}$, breaking up from the top of the hump with the initial speed $\boldsymbol{\nu}_{\mathbf{0}}$, can be converted into potential energy $\boldsymbol{E}_{\mathbf{p}}$ of wagon, which is waiting for its break thrust on the top of the hump, located at a height of $\boldsymbol{h}_{\text {hump, and vice }}$ versa.

Mechanical system "wagon - track rail" described in the solution of engineering problems in [16], is a proprietary system, i.e. a system with non-ideal constraints, where part of the kinetic energy acquired by the wagon after the breaking up of the hump crest, lost in overcoming the work of the forces of resistance of any kind (friction forces; forces of resistance that appears when the passage curves plots the path; the forces of resistance that occur when hitting the blades of switches; the drag force from the aerial environment and of wind, of snow and frost).

In this regard, the hypothesis adopted in [16] that the total energy is spent on overcoming the work of the forces of any resistance (see mathematical expression (4)), contradicts the theorem on the change of kinetic energy for a non-free material point in the final form $[6,19]$, where the work of the resistance force must have a negative sign:

$$
\Delta E=-A_{\mathrm{r}, \mathrm{c}, \mathrm{s}}
$$

Hence the required height of the hump $\boldsymbol{h}_{\text {hump }}$ can be defined as, m:

$$
h_{\text {hump }}=-\frac{1}{g M} A_{\mathrm{r}, \mathrm{c}, \mathrm{s}}-\frac{v_{0}^{2}}{2 g^{\prime \prime}}
$$

As can be seen, the obtained formula is not comparable with formula (9), since the result is pseudoscientific.

Thus, analyzing the results of [16], we note that they contradict the theorem on the change of kinetic energy for a nonfree material point in the final form. From here it becomes obvious the relevance of discussion of theoretical provisions on the choice of height of the first profile site (from the top of the hump to the first switching area or the first brake position (1BP)) of the marshalling hump.

\section{Object}

Object:

- mathematically prove the absence of a theoretical basis for the formula by which to calculate the height of the first profile section of the marshalling hump;

- the results of the calculations to justify and / or refute the correctness of the derivation of the formula for calculating the height of the first profile section (from the top of the hump to the braking zone of the first brake position) of hump; 
- to justify the erroneous use in the normative-technical document of the formula $h_{0}=$ $v_{0}^{2} / 2 g^{\prime}$ to determine the energy height of the hump taking into account the inertia of the rotating parts, applicable only for the ideal connection (see pp. 286, 287 in [19]).

\subsection{Research methods}

The research methods are based on the use of the theorem on the kinetic energy change for a non-free material point in the final form $[6,19]$.

\subsection{The main findings of the study}

To determine the height of the first section of the profile hump $\boldsymbol{h}_{\mathbf{1}}$, applying the theorem on the change of kinetic energy for a free material point (which means that no restrictions are imposed on its movements) in a finite form $[6,19]$ we obtain:

$$
\frac{G}{2 g_{o}^{\prime}} v_{\mathrm{in}}^{2}-\frac{G}{2 g_{o}^{\prime}} v_{0}^{2}=A_{G}
$$

where $\boldsymbol{A}_{\boldsymbol{G}}$ - the work of gravity $\boldsymbol{G}$, as a potential force that maintains a constant direction (vertical down) and modulus:

$$
A_{G}=G h_{1}
$$

given the fact that it $\boldsymbol{h}_{\mathbf{1}}$ - vertical movement of the body from gravity $\boldsymbol{G}$, otherwise, according to formula (1), part of the height of the first profile section (from the top of the hill to the first arrow zone or the first brake position), $\mathrm{m}$.

Further, substituting (14) into (13), after elementary calculations we obtain:

$$
h_{1}=\frac{v_{\mathrm{in}}^{2}-v_{0}^{2}}{2 g_{o}^{\prime}} .
$$

Hence, it is clear that the last formula is derived correctly, however, considering the connection (profile section of the hump) ideal (smooth and/or frictionless).

It is obvious that by formula (15) it is permissible to calculate the part of the height of the first profile section of the hump $\boldsymbol{h}_{\mathbf{1}}$, assuming that this section is an ideal (smooth and/or frictionless) connection. However, in reality, the profile of this section of the slide corresponds to a non-ideal (non-smooth and/or friction) connection (i.e. track rail).

In this regard, it is not clear and / or doubtful for what reason the dependence is still widely used to determine the energy heights in the design methodology of sorting slides (see the fifth cotrprimer in [1]) relation is still widely used $\boldsymbol{h}=\boldsymbol{f}\left(\left[\boldsymbol{v}_{\mathrm{in}}\right], \boldsymbol{v}_{\mathbf{0}}, 2 \mathrm{~g}^{\prime}\right)$ (where [ $\left.\boldsymbol{v}_{\mathrm{in}}\right]-$ the permissible input speed of the estimated free axle the retarders 1TP under favorable conditions, sliding, $\mathrm{m} / \mathrm{s} ; \boldsymbol{v}_{\mathbf{0}}$ - the greatest initial velocity sliding FA, m/s), arising from formula (1) in [6] where $\boldsymbol{a}=g^{\prime} i=g^{\prime} h / l$ (see formula (7) in [15]), fair only for ideal connections (see p. 287 in [19]).

Discuss the second term of formula (1). Specific energy losses $\boldsymbol{h}_{\mathbf{s p 1}}$, as the specific work of the forces of resistance to movement, is determined by the formula (14) in [12]:

$$
h_{\mathrm{sp} 1}=l_{1} \omega_{0} 10^{-3}
$$

where $\omega_{0}$ - the calculated value of the basic resistivity of the movement, taken in [12] at calculation of height of the hill depending on weight category of cars, and at constructive 
and technological calculations - from type of the runner (for example, GA or GFA) and conditions of rolling (favorable or unfavorable), $\mathrm{kgf} / \mathrm{ts}$; $\boldsymbol{l}_{\mathbf{1}}$ - the length of the section of the track on which the effect of the main resistance is considered $\boldsymbol{\omega}_{\mathbf{0}}$, as an imperfect connection, $m$.

Analyzing the formula (16), we note that in it the product of the length of the track section $\boldsymbol{l}_{\mathbf{1}}$ (in $\mathrm{m}$ ) on the basic resistivity to movement $\boldsymbol{\omega}_{\mathbf{0}}$ (в $\left.\mathrm{kgf} / \mathrm{tf}\right)$, how different the meaning of physical concepts (measure of length and the measure of the imperfect communication in non-systemic units of measurement), does not fit into the basic principles of General mechanics [19], to which attention is drawn also in the twelfth counterexample in [12].

As can be seen, the formula (16) cannot be derived using the principles and/or methods of theoretical and/or engineering mechanics [6, 20], and therefore has no theoretical basis.

We also discuss the content of the third term of formula (1). Loss of specific energy when overcoming resistance from switches and curves $\boldsymbol{h}_{\text {ac1 }}$ within the advance section $\boldsymbol{l}_{\mathbf{1}}$ :

$$
h_{\mathrm{ac} 1}=\left(0.56 n+0.23 \sum \alpha_{\mathrm{ac}}\right) v^{2} 10^{-3},
$$

where $\boldsymbol{n}$ - number of switches; $\sum \boldsymbol{\alpha}_{\mathbf{a c}}-$ sum of angles of rotation, deg., including arrow angles, on the route (section) of rolling [16]; $\boldsymbol{v}$ - the average speed of the wagon on the route (section) of rolling, as a normalized value, i.e. $v=\left[v_{\mathrm{m}}\right], \mathrm{m} / \mathrm{s}$.

In the last formula $\boldsymbol{n}$ and $\sum \boldsymbol{\alpha}_{\mathbf{a c}}$ find from the plan of a neck of marshalling tracks (see $\mathrm{p}$. 141 in [13]). Analysis of the formula (17) shows that it is empirical, has no theoretical basis, and, at the same time, it is determined $\boldsymbol{h}_{\text {ac1 }}$ depending on the average speed of the car on the route (section) rolling, i.e. $\boldsymbol{h}_{\mathrm{ac}}=\boldsymbol{f}\left(\boldsymbol{n}, \sum \boldsymbol{\alpha}_{\mathrm{ac}},\left[\boldsymbol{v}_{\mathrm{m}}\right]\right)$.

Here bewilderment and/or doubt is that only at the initial stage of calculation of parameters of a hump use $\boldsymbol{v}=\left[\boldsymbol{v}_{\mathbf{m}}\right]$, the magnitude of which and the permissible value of the speed of the entrance of the car to the retarders $\boldsymbol{v}_{\text {in }}=\left[\boldsymbol{v}_{\mathbf{r m}}\right]$, without performing any other calculations, you can find the sought speed, for example, at the end of the speed section $\boldsymbol{v}_{\mathbf{s s} 2}$.

Generalizing the analysis of formulas (16) and (17) containing elements of non-ideal connection in the form $\boldsymbol{\omega}_{\mathbf{0}}, \boldsymbol{n}$ and $\sum \boldsymbol{\alpha}_{\mathbf{a c}}$, it can be argued that they have no theoretical basis, and formula (15) holds for an ideal connection. For this reason, in contrast to the erroneous opinion of the authors of the article [11] (see the first paragraph of the last column on page 36 in [14]), it is according to the formula (2) in [14], slide structural and technological calculations modeling the conditions of movement of calculated free axles with different running properties can not be performed.

\section{Research result}

\subsection{Calculation example.}

For example, let us perform a comparative calculation of the height of the first profile section (from the top of the hump to the braking zone of the first brake position) of the hump according to the formula (1), comparing the result with the data performed by the exact formula $\boldsymbol{h}=\boldsymbol{l} \cdot \boldsymbol{i}$ (see formula (4) in [15]) for each section. Here we take into account that the first profile section consists of the first and second high-speed sections $\left(\boldsymbol{l}_{1}, \boldsymbol{l}_{\mathbf{2}}\right)$ and the length of the wagon wheelbase $\left(\boldsymbol{l}_{\text {in1 }}\right)$.

The initial data of the example are as follows: $\boldsymbol{l}_{\mathbf{1}}=39.95, \boldsymbol{l}_{\mathbf{2}}=15.007, \boldsymbol{l}_{\mathbf{2}}=18.633$ и $\boldsymbol{l}_{\mathbf{i n} 1}=$ 8.301 - length of the projected sections of the hump, $\mathrm{m} ; \boldsymbol{i}_{\mathbf{1}}=50, \boldsymbol{i}_{\mathbf{2}}=30, \boldsymbol{i}_{\mathbf{2} \mathbf{c}}=18$ and $\boldsymbol{i}_{\mathbf{B x} \mathbf{1}}=$ 14 - slope of the studied areas, $\%$; $\boldsymbol{v}_{\text {in }}=7.924$ - the maximum permissible input speed of 
single cars on a railway car retarders (which is less than the allowable input speed of the car retarders of the type KZ-3, KZ-5 and VSGP-5 [20]), m/s; $\boldsymbol{v}_{\mathbf{0}}=1.7$ - highest initial rolling speed " of a very good free axle (GFA)», $\mathrm{m} / \mathrm{s} ; g_{0}^{\prime}=9,635$ - acceleration of gravity taking into account the inertia of the rotating masses GFA for $\boldsymbol{G}=908 \mathrm{\kappa H}, \mathrm{m} / \mathrm{s}^{2}$.

\subsection{Calculation results.}

We calculate part of the height of the first profile section of the hump $\boldsymbol{h}_{\mathbf{1}}$. by formula (15), derived under the assumption that the profile section of the hump is an ideal (smooth and/or frictionless) bond, $\mathrm{m}$ :

$$
h_{1 \mathrm{ps} .}=\frac{v_{\mathrm{in}}^{2}-v_{0}^{2}}{2 g_{\mathrm{o}}^{\prime}}=\frac{7.924^{2}-1.7^{2}}{2 \cdot 9.635}=3.108 .
$$

We calculate, according to the generally accepted understanding (see page 36 in [11]), the loss of specific energy when overcoming the basic specific resistance to motion $\boldsymbol{h}_{\text {sp1 }}$ within the advance section $\boldsymbol{l}_{\mathbf{1}}$ by formula (16), $\mathrm{m}$ :

$$
h_{\mathrm{sp} 1}=l_{1} \omega_{0} 10^{-3}=81.891 \cdot 0.5 \cdot 10^{-3}=0.041 .
$$

We calculate the loss of specific energy when overcoming the resistance of the arrows and curves $\boldsymbol{h}_{\text {ac1 }}$ within the advance section $\boldsymbol{l}_{\mathbf{1}}$ by formula (17), m:

$$
\begin{gathered}
h_{\mathrm{ac} 1}=\left(0.56 n+0.23 \sum \alpha_{\mathrm{ac}}\right) v^{2} 10^{-3}= \\
=(0.56 \cdot 1+0.23 \cdot 0.083) \cdot 4.5^{2} 10^{-3}=0.012 .
\end{gathered}
$$

The height of the first profile section (from the hump apex to the braking zone of the first brake position (1BP)) of the hump is calculated by equation (1), m:

$$
h_{1}^{\max }=\frac{v_{\mathrm{in}}^{2}-v_{0}^{2}}{2 g_{\mathrm{o}}^{\prime}}+h_{\mathrm{sp} 1}+h_{\mathrm{ac} 1}=3.108+0.041+0.012=3.161 .
$$

Disregard values $\boldsymbol{h}_{\text {sp1 }}$ and $\boldsymbol{h}_{\text {ac1 }}$ obvious, since $\boldsymbol{h}_{\mathbf{1 p s} .} \gg>\left(\boldsymbol{h}_{\mathbf{s p} \mathbf{1}}+\boldsymbol{h}_{\mathbf{a c} \mathbf{1}}\right)$, i.e. $3.108>>(0.041+$ $0.012)=0.053 \mathrm{~m}$.

Perform the height calculation $\boldsymbol{h}_{\mathbf{1}}$ the first profile section of the hump according to the initial length data $\left(\boldsymbol{l}_{\mathbf{1}}, \boldsymbol{l}_{2}, \boldsymbol{l}_{\mathbf{2}}, \boldsymbol{l}_{\text {in1 }}\right)$ and slope $\left(\boldsymbol{i}_{1}, \boldsymbol{i}_{2}, \boldsymbol{i}_{2 v}, \boldsymbol{i}_{\text {in1 }}\right)$ these areas by the exact formula (4) $\boldsymbol{h}_{\boldsymbol{b r i}}=\boldsymbol{l}_{\boldsymbol{b r i}} \boldsymbol{i}_{\boldsymbol{b} r \boldsymbol{i}}$, in [12]), e.g., $\boldsymbol{h}_{\mathbf{1}}=\boldsymbol{l}_{\mathbf{1}} \cdot \boldsymbol{i}_{1}, \mathrm{~m}$ :

$$
h_{1 \mathrm{br}}=h_{1}+h_{2}+h_{2 v}+h_{\text {in } 1}=1.994+0.45+0.335+8.301=2.595 \text {. }
$$

As can be seen, the height of the first profile section of the hump, calculated by the formula (1) and the exact formula of the form $\boldsymbol{h}_{b r i}=\boldsymbol{l}_{\boldsymbol{b r i}} \boldsymbol{i}_{\boldsymbol{b} r \boldsymbol{i}}$ (see formula (4) in [15]) has relative error $\boldsymbol{\delta} \boldsymbol{h}=17,9 \approx 18 \%$, not acceptable for engineering calculations.

We assume that such results are caused by the fact that part of the height of the first profile section of the hump $\boldsymbol{h}_{\mathbf{1 p s}}$ it is calculated by the formula (1), derived under the assumption that the profile section of the hump is an ideal connection, and the loss of specific energy when overcoming the basic specific resistance to movement $\boldsymbol{h}_{\text {sp1 }}$ and resistance from switches and curve line $\boldsymbol{h}_{\text {sp1 }}$ within the advance section $\boldsymbol{l}_{\mathbf{1}}$ - by analytically unprovable empirical formulas for non-ideal connections (track rail).

Thus, summarizing the results of checking the correctness of the derivation of the formula (1) to calculate the height of the first profile section (from the top of the slide to the braking zone of the first brake position) slides in comparison with the results of the calculated data, it can be concluded that in the formula (1) summed values obtained for obviously different conditions. For this reason, they contain undeniable errors bearing 
elements of pseudoscientific material. Here we make a reservation that similar to formulas (1), (16) and (17) causes objections relating to the determination of the height of the hump.

The formula for determining the speed of the wagon at any given point in the profile of the hump [12], used only to construct the curves of the speed of free axles, and finding the specific energy corresponding to the established rate of breaking-up, can also be criticized $v_{\mathbf{0}}=\boldsymbol{v}_{\mathbf{o d}}$, according to Galileo's formula $(3)\left(v=\sqrt{2 g h}\right.$ and $\left.h_{0}=v_{0}^{2} / 2 g^{\prime}[12,13]\right)$.

Especially stipulate that the formula $h_{0}=v_{0}^{2} / 2 g^{\prime}$ in $[12,13]$ to determine the energy height of the slide, taking into account the inertia of the rotating parts, it is applicable only for ideal communication (see pages 286, 287 in [19]) and in no case can it be used to determine the sliding speed of the car in the braking zones in the sections of the braking positions. Otherwise, there will be an unscientific result. Note that $h_{0}=v_{0}^{2} / 2 g^{\prime}$ in $[12,13]$ derived from Galileo's formula $v=\sqrt{2 g h}$ [15] to determine the rate of free fall of object. Recall that track rails can be considered as ideal bonds only under the assumption that they are absolutely solid. Unfortunately, there are no such objects in nature.

As can be seen, the formula (8) in [12] to determine the height of the first profile section (from the top of the hump to the first pointer zone or the first brake position), which is widely used in the design of hump, as one of the main criticisms of the authors [12], for some unknown reason, instead of conducting serious scientific analysis, explanations and theoretical justifications, unfortunately, remained without attention of authors [13, 14].

In this regard, summarizing the results of the critical analysis of the formula (1), it can be argued that in it, the first term is correct only for the ideal connection, and the second and third terms - for non-ideal connection, which indicates its incorrectness and, for this reason, the inadmissibility of the use in the methodology. Otherwise, the combination of terms in one formula is erroneous, where one of them corresponds to an ideal connection, and the other two correspond to a non-ideal connection.

Let us make a special reservation that the correct solution of the problem of moving the wagon down the profile of the marshalling hump from its top to the calculated point is given in $[6,20]$.

\subsection{Application area for results}

The results of the research can be used in the processing of normative and technical document on the design of marshalling devices on railways and making adjustments to the position of the dynamics of rolling the wagon down the profile of the marshalling hump in textbooks for universities of railway transport.

\section{Conclusions}

1. Analyzing the results of [16], it is revealed that the formula by which the height of the first profile section of the marshalling hump is calculated (see formula (8) in [12]) contradicts the theorem on the change of kinetic energy for a non-free material point in the final form.

2. The results of the calculations proved incorrect derivation of the formula for calculating the height of the first profile section of the hump in the normative and technical document "Rules and regulations for the design of marshalling devices on railways with gauge 1520 mm". 
3. The error of use of formula $h_{0}=v_{0}^{2} / 2 g^{\prime}$ in the normative-technical document is proved to determine the energy height of the slide taking into account the inertia of the rotating parts, applicable only for the ideal connection (see pp. 286, 287 in [19]).

\section{References}

1. J. Prokop, Sh. Myojin, Yard. Memoirs of the Faculty of Engineering 27(2), 59-71 (1993).

2. C. Zhang, Y. Wei, G. Xiao, Z. Wang, J. Fu, Proc. of Second Intern. Conf. on Transportation and Traffic Studies, 285-290 (2000). DOI: 10.1061/40503(277)45.

3. S. Zářecký, J. Grúň, J. Žilka, Transport Problems 3(4), 87-95, (2008).

4. T. Judge, Railway Age, 5, 33-34 (2007)

5. C. Zhang, Y. Li, Proc. of Intern Conf. «Optoelectronics and Image Processing (ICOIP)», 2, 262-265 (2010). DOI: 10.1109/ICOIP.2010.274.

6. K.L. Komarov, A.F. Yashin, Teoreticheskaya mekhanika $v$ zadachah zheleznodorozhnogo transporta (Theoretical mechanics in railway transport problems), 296 (Nauka, Novosibirsk, 2004)

7. V. Bobrovskyi, D. Kozachenko, A. Dorosh, E. Demchenko, T. Bolvanovska, A. Kolesnik, Transport Problems. IV Symposium of Young Researchers, 632-640, (2015)

8. V. Bobrovskyi, D. Kozachenko, A. Dorosh, E. Demchenko, T. Bolvanovska, A. Kolesnik, Transport Problems 11(1), 147-155 (2016). DOI: 10.20858/tp.2016.11.1.14.

9. D.M. Kozschenko, V.I. Bobrovsky, S.V. Grevtsov, M.I. Berezobyi. Science and transport progress. Bulletin of Dnipropetrovsk National University of Railway Transport 3(63), 28-40 (2016). DOI:10.15802/stp2016/74710.

10. D. Kozachenko, V. Bobrovskyi, Y. Demchenko, Journal of Modern Transportation 26(3), 189-199 (2018). DOI:10.1007/s40534-018-0161-2

11. Kh. T. Turanov, A. Gordiienko, Open Access Library Journal 2, e1912, 1-11, (2015). doi: http://dx.doi.org/10.4236/oalib.1101912. PP.1-11.

12. Kh. T. Turanov, Transport information Bulletin 3(237), 29-36 (2015)

13. V.M. Rudanovskij, I.P. Starshov, Transport information Bulletin 6(252), 19-28 (2016)

14. Yu.O. Pozojskij, V.A. Kobzev, I.P. Starshov, V.M. Rudanovskij, Transport information Bulletin 2, 272, 35-38 (2018)

15. Kh.T. Turanov, A.A. Gordienko, Transport information Bulletin 9(279), 23-28 (2018)

16. J. Prokop, Sh. Myojin, Memoirs of the Faculty of Engineering 27(2), 41-58 (1993).

17. Kh.T. Turanov, Gordienko, A., MATEC Web of Conferences 216, 02027 (2018)

18. Kh.T. Turanov, E. Timukhina, A. Gordienko, TransSiberia, 703-716 (2019). DOI:10.1007/978-3-030-37916-2_69

19. S.P. Timoshenko, Inzhenernaya mekhanika (Engineering mechanics), 507 (Mashgiz, Moscow, 1960)

20. V.A. Kobzev, Technical means of marshalling humps, ensuring traffic safety. Part 1. Textbook (Tekhnicheskie sredstva sortirovochnyh gorok, obespechivayushchie bezopasnost' dvizheniya), 92 (MIIT, Moscow, 2009) 